\title{
STOMACH
}

\section{Inflammation and intestinal metaplasia at the squamocolumnar junction in young patients with or without Helicobacter pylori infection}

\author{
A Oksanen, P Sipponen, R Karttunen, H Rautelin
}

See end of article for authors' affiliations

Correspondence to: Dr A Oksanen, Herttoniemi Hospital, PL 6300, FIN-00099 Helsinki, Finland;

aino.oksanen@hel.fi

Accepted for publication 3 September 2002
Background: Intestinal metaplasia (IM) in the oesophagus is a known risk factor for adenocarcinoma of the oesophagus. The incidence of adenocarcinoma of the cardia and oesophagus has increased in Western countries simultaneously with a decrease in Helicobacter pylori prevalence.

Aims: To determine the association of $H$ pylori infection with inflammation and IM at the squamocolumnar junction (SCJ) in young individuals.

Patients: A total of 168 (121 women; $72 \%$ ) consecutive outpatients, $\leqslant 45$ years, undergoing gastroscopy, and with no prior $H$ pylori eradication treatment.

Methods: Biopsy specimens taken from the antrum, corpus, SCJ, and oesophagus were assessed according to the updated Sydney system, and type of IM (complete or incomplete) was determined. Serum samples from $\mathrm{H}$ pylori positive patients were studied for CagA antibodies.

Results: In $86 \%$ of 37 patients with gastritis in the antrum and/or corpus (24 histologically $H$ pylori positive) and in $23 \%$ of 125 patients with a healthy stomach, inflammation was present in the glandular mucosa at the SCJ. In the latter, cardiac mucosa more often than fundic mucosa at the SCJ was inflamed $(p<0.001)$, the inflammation was usually milder in nature, and was associated with signs of reflux disease. IM (incomplete or complete) at the SCJ was evident in nine of those 24 with a healthy stomach and inflamed cardiac mucosa at the SCJ but in none of those with $\mathrm{H}$ pylori gastritis.

Conclusions: IM at the SCJ can also appear in young individuals in whom it seems to be associated with reflux related isolated inflammation in cardiac mucosa at the SCJ but not with $\mathrm{H}$ pylori gastritis.
$\mathrm{T}$ he presence of Helicobacter pylori infection, especially that caused by a CagA positive strain, appears to be negatively associated with Barrett's oesophagus, ${ }^{1}$ dysplasia in Barrett's oesophagus, ${ }^{12}$ and adenocarcinoma of the cardia and oesophagus. ${ }^{1-4}$ This has raised a question as to a possible causal relationship between the increase in incidence of adenocarcinoma of the cardia and oesophagus and a simultaneous decrease in $H$ pylori prevalence in Western countries. ${ }^{5}$

A columnar epithelium lined tubular oesophagus $2-3 \mathrm{~cm}$ in length with incomplete intestinal metaplasia (IM) is a well known risk factor for adenocarcinoma of the oesophagus. This classic Barrett's oesophagus is however a relatively uncommon finding, detected in only $1-2 \%$ of patients undergoing gastroscopy. ${ }^{6-8}$ Subsequently, only $2-6 \%$ of oesophageal adenocarcinomas have been reported to occur in patients with known Barrett's oesophagus. ${ }^{10}$ In patients without classic Barrett's oesophagus, adenocarcinomas of the gastrooesophageal junction appear to arise from foci of IM at the squamocolumnar junction (SCJ), ${ }^{11}$ which occur in $9-36 \%$ of patients undergoing gastroscopy. ${ }^{6}{ }^{12-14} \mathrm{IM}$ at the SCJ or in the cardia has however been shown to be associated with $H$ pylori infection. ${ }^{15} 16$

If acquired, lesions at the SCJ may be related to time of exposure to possible risk factors and to patient age; early lesions may thus be assumed to be present in young individuals in particular. This makes findings in young individuals especially interesting. The present study explored the association of $H$ pylori infection with inflammation and IM at the SCJ in young individuals.

\section{PATIENTS AND METHODS}

Consecutive Caucasian outpatients, $\leqslant 45$ years, with no prior $H$ pylori eradication treatment undergoing gastroscopy at
Herttoniemi Municipal Hospital between March 1998 and July 1999 were included, many of whom also took part in a study assessing a serological rapid test for $H$ pylori in a basic endoscopy population. ${ }^{17}$ The study was approved by the ethics committee of the Helsinki City Health Department.

The study population originally comprised 172 patients, $\leqslant 45$ years, referred for gastroscopy, although for four, biopsies from the columnar side of the SCJ were unavailable, excluding these patients from further analyses. Median age of the remaining 168 patients was 34 years: 36 were 18-25 years, 60 were $26-35$ years, and 72 were $36-45$ years; 121 (72\%) were women. The indication for gastroscopy was heartburn and/or regurgitation in 65 patients, dyspepsia or upper abdominal pain in 46 , suspicion of coeliac disease in 38 , follow up of coeliac disease in five and of atrophic gastritis in three, and miscellaneous reasons in 11 .

A routine gastroscopy was performed by one author (AO) with an Olympus GIF-Q140 videoendoscope (Olympus Finland, Helsinki, Finland). The SCJ was assessed visually. Distances were measured from the diaphragmatic hiatus and from the SCJ to the bite block. The diaphragmatic hiatus was identified as the narrowest portion of the distal oesophagus and, in the case of hiatal hernia, as the narrowest level of the junction between the stomach and hiatal hernia sac. Hiatal hernia was defined as the combination of a wide hiatal opening when assessed with a retroflexed gastroscope, and a distance of at least $2 \mathrm{~cm}$ between the diaphragmatic hiatus and the SCJ. Erosive oesophagitis, defined as any erosions

Abbreviations: GORD, gastro-oesophageal reflux disease; IM, intestinal metaplasia; SCJ, squamocolumnar junction. 

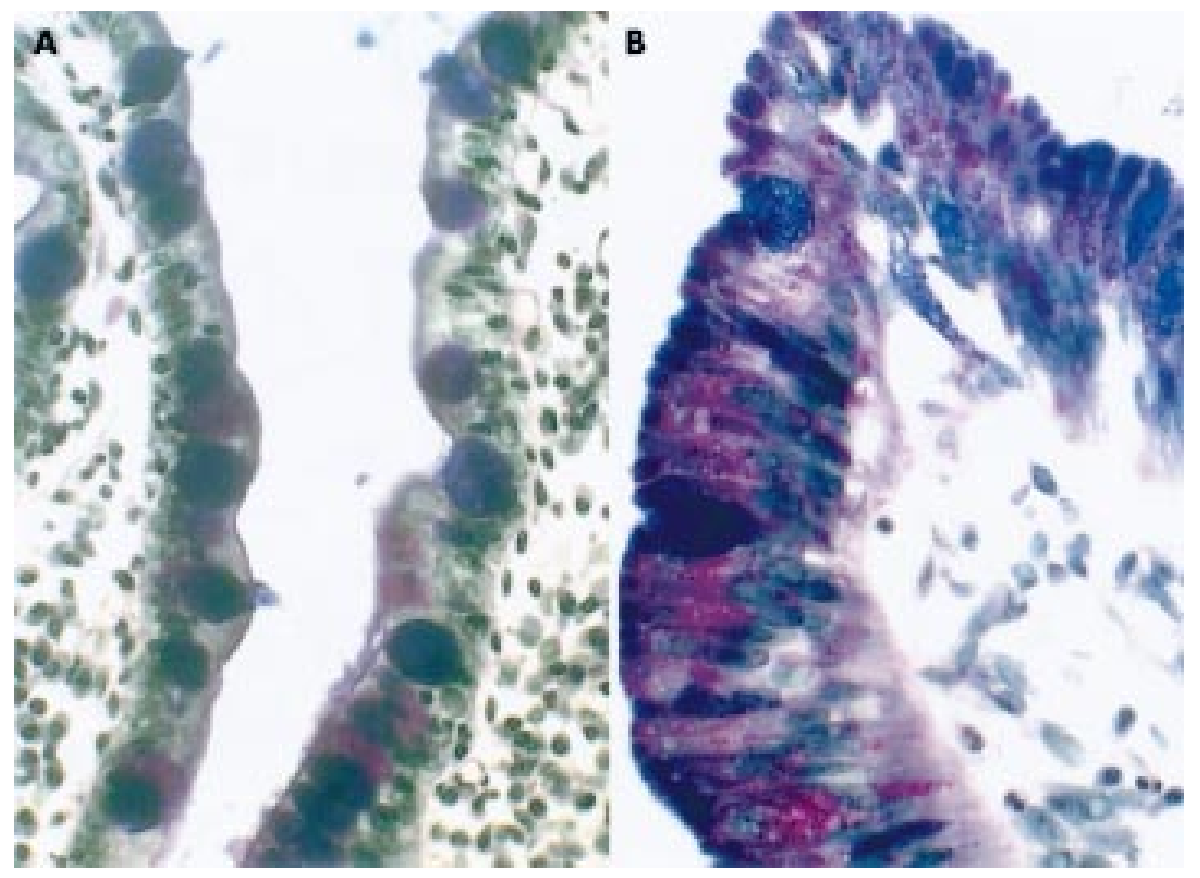

Figure 1 Complete $(\mathrm{A})$ and incomplete (B) intestinal metaplasia at the squamocolumnar junction, stained by alcian blue (pH2.5)-periodic acid-Sciff. seen on the squamous epithelium, was classified according to the Los Angeles (LA) classification. ${ }^{18}$

All biopsy specimens were obtained with standard biopsy forceps. In addition to the two biopsy specimens each taken from the antrum and corpus, one to two specimens (or in some cases even more) were taken from the glandular mucosa at the SCJ or within $5 \mathrm{~mm}$ from the SCJ, with the endoscope in the anterograde position. One biopsy specimen came from the squamous epithelium of the oesophagus about $1 \mathrm{~cm}$ above the SCJ.

Biopsy specimens were stained with haematoxylin-eosin, alcian blue ( $\mathrm{pH}$ 2.5)-periodic acid-Schiff, and modified Giemsa stains, and assessed for the presence of gastritis, $H$ pylori, and metaplasia in a blinded fashion by one pathologist (PS) and scored in accordance with the updated Sydney system. ${ }^{19}$ Histological oesophagitis was defined as basal cell hyperplasia and papillary elongation or infiltration of inflammatory cells in the squamous mucosa. For all biopsy specimens from the SCJ, mucosal type (antral, fundic, cardiac, squamous) was recorded. Cardiac mucosa was defined as mucosa with mucin secreting glands in which some single parietal cells might be present but without the appearance of normal fundic mucosa. Biopsy specimens from the SCJ were assessed for the presence and type of IM, IM defined as glandular mucosa with non-equivocal goblet cells. In complete IM, the non-goblet columnar cells were absorbing and not mucin secreting cells, while in incomplete IM the non-goblet cells contained neutral and acid mucins (fig 1). In some patients, both types of IM were present; these were recorded as being in the group with incomplete IM.

For 22 of the 24 histologically $H$ pylori positive patients, serum samples were available and were tested for CagA antibodies by a commercial immunoblot method (ID Blot $H$ pylori IgG; DPC, Los Angeles, California, USA) according to the manufacturer's protocol. Briefly, serum samples at a dilution of 1:50 were incubated with nitrocellulose strips for 30 minutes at room temperature. The strips were washed with buffer, incubated with an enzyme labelled anti-IgG antibody and, after washing, treated with a solution containing a BCIP (5-bromo-4-chloro-3-indolyl phosphate) substrate. Positive bands containing anti-CagA antibody were estimated visually.

Fisher's exact test and the $\chi^{2}$ test were used, when appropriate, to compare the proportions of various characteristics between groups. The Jonckheere-Terpstra test was used to compare grades of inflammation. A p value of $<0.05$ was considered significant.

\section{RESULTS}

\section{Endoscopic findings}

Macroscopical findings included hiatal hernia (56 patients), erosive oesophagitis (12, LA class A-B), gastric ulcer (3), and duodenal ulcer (2). No patient had classic Barrett's oesophagus. In one patient, the SCJ was located $<1 \mathrm{~cm}$ above the hiatus hernia sac in the tubular oesophagus (thus he had a short segment Barrett's oesophagus) but in all others the SCJ was normally located. Four patients had tongues of columnar mucosa $>5 \mathrm{~mm}$ in length, 36 patients had tiny tongues $(<5$ $\mathrm{mm}$ ) or tortuous SCJ, and in 43 additional patients the SCJ seemed somewhat irritated (irregular). In 84 patients, the SCJ looked normal.

\section{Histology of gastric mucosa}

Among the patients, 125 (74\%) had healthy stomachs-that is, histological findings in the biopsy specimens from the antrum and corpus were entirely normal-whereas 37 (22\%) had gastritis in the antrum, the corpus, or both. Biopsy specimens from the antrum and corpus in the remaining six showed mild atrophic changes or IM without significant gastritis. Of the 37 with gastritis, 24 (14.3\% of all 168) were histologically positive for $H$ pylori. Of the 13 patients with $H$ pylori negative gastritis, six had an autoimmune type of corpus predominant atrophic gastritis (all women), five had Crohn-like gastritis, one had interstitial gastritis representing findings of lymphocytic gastritis, and one had mild reactive gastritis in the antrum and a normal corpus. Only one patient with $H$ pylori gastritis had IM in the distal stomach (in the antrum). All six with the autoimmune type of atrophic corpus gastritis had IM in the corpus, and one in the antrum also.

\section{Histology of biopsy specimens from the SCJ}

Biopsies of the columnar side of the SCJ showed cardiac mucosa in 83 patients and only fundic mucosa in 84. Biopsy specimens in one patient were too superficial for definition of their glandular status. In 33 patients $(20 \%)$ the squamous to columnar mucosal interface was present in at least one biopsy specimen; none had true corpus-type mucosa adjacent to squamous epithelium. 
Table 1 Grade of chronic inflammation in glandular mucosa at the squamocolumnar junction (SCJ) in patients with Helicobacter pylori gastritis and in those with a healthy stomach. Grade of active inflammation, if present, is also shown

\begin{tabular}{lll}
\hline & \multicolumn{2}{l}{$\begin{array}{l}\text { No of patients with chronic inflammation at } \\
\text { the SCJ and: }\end{array}$} \\
\cline { 2 - 3 } Grade of & $\begin{array}{l}\text { H pylori gastritis } \\
(\mathrm{n}=23)\end{array}$ & $\begin{array}{l}\text { Healthy stomach } \\
(\mathrm{n}=29)\end{array}$ \\
\hline Chronic inflammation & & \\
$\quad$ Mild & 2 & 24 \\
$\quad$ Moderate & 12 & 5 \\
Severe & 9 & 0 \\
Active inflammation & & \\
$\quad$ None & 8 & 21 \\
Mild & 14 & 7 \\
Moderate & 0 & 1 \\
Severe & 1 & 0 \\
\hline
\end{tabular}

For 150 patients, adequate biopsy specimens were available from squamous mucosa of the oesophagus; 30 of these (20\%) had histological oesophagitis. Hyperplastic changes occurred in the columnar mucosa at the SCJ in 11 patients with cardiac mucosa and in one patient with fundic mucosa; six of these had inflamed mucosa.

\section{Inflammation at the SCJ}

Of the 168 patients, 64 (38\%) had chronic inflammation in the glandular mucosa at the SCJ. In the entire study group, such inflammation was associated with gastritis in the antrum or corpus or both $(\mathrm{p}<0.001)$ and with $H$ pylori positivity $(\mathrm{p}<0.001)$, but not with histological or endoscopic oesophagitis.

Of the 37 patients with distal gastritis, 24/26 with cardiac mucosa and 8/10 with fundic mucosa at the SCJ had inflammation in glandular mucosa at the SCJ. For $1 / 37$ only a superficial specimen was available. In 23/24 patients histologically positive for $H$ pylori, inflammation was present in the glandular mucosa at the SCJ, and in 21, helicobacters appeared at this site also. Of the 22 histologically $H$ pylori positive patients with serum samples available, $18(82 \%)$ were positive for CagA antibodies.

Among the 125 patients with a healthy stomach, 24/54 of those with cardiac mucosa and 5/71 of those with only fundic mucosa in biopsy specimens from the SCJ had inflammation at the SCJ $(p<0.001)$. Five patients with several biopsy specimens from the SCJ showed both inflamed cardiac mucosa and uninflamed fundic mucosa. Among the 125, inflammation was present at the SCJ in $23 \%$, and was associated with histological $(p=0.0127)$ and endoscopic $(p=0.0409)$ oesophagitis and with hiatal hernia $(p=0.0098)$ but not with reflux symptoms as the indication for gastroscopy.

Grades of both chronic $(p<0.0001)$ and active $(p=0.0088)$ inflammation at the SCJ were higher in patients with multifocal $H$ pylori gastritis than in patients with a healthy stomach and isolated SCJ inflammation (table 1). In patients with $H$ pylori gastritis, grades of inflammation were similar in cardiac and fundic mucosa. Among those with a healthy stomach, inflammation in fundic mucosa at the SCJ was always mild (grade 1) and never active.

\section{Intestinal metaplasia at the SCJ}

IM at the SCJ was found in $11 / 168(6.5 \%)$ patients, with metaplasia of the incomplete type in six (3.5\%) (table 2). Two patients with $H$ pylori negative gastritis had IM at the SCJ. All other patients with IM at the SCJ had a healthy stomach and none had $H$ pylori infection. IM was never seen in a biopsy specimen which included fundic mucosa. In all patients with IM at the SCJ, inflammation also occurred at this site. Two patients had extensive IM with more than half the specimen infiltrated by goblet cells (Nos 3 and 11 in table 2); in others, the goblet cells only infiltrated less than half of the specimen. No patient had dysplasia in these or any other specimens.

IM at the SCJ was more common in men $(5 / 48,10 \%)$ than in women $(6 / 121,5 \%)$; this however was not statistically significant. IM at the SCJ was associated with SCJs showing tongues or being tortuous $(p=0.005)$, but no significant association existed between IM at the SCJ and the presence of IM in the distal stomach, endoscopic or histological oesophagitis, hiatus hernia, or reflux symptoms as the indication for gastroscopy.

\section{DISCUSSION}

This study showed that patients with inflammation at the SCJ could be divided into two main groups. One group included patients with antral/fundal gastritis and in most cases positive $H$ pylori and CagA status; of these, $86 \%$ had inflammation at the SCJ also. Nearly all patients with distal $H$ pylori gastritis had $H$ pylori organisms at the SCJ, implicating the organism as a possible cause of inflammation at their SCJs also. The second group comprised patients with a healthy stomach, of whom $23 \%$ had inflammation at the SCJ, and this inflammation was associated with histological and endoscopic oesophagitis, and hiatal hernia. Similar findings have been reported in two other studies in which inflammation at the SCJ was associated with $H$ pylori gastritis and, in $H$ pylori negative patients, with gastro-oesophageal reflux disease (GORD) ${ }^{20}{ }^{21}$ In these earlier studies assessing patients of all ages with a normal appearing

Table 2 Characteristics of patients with incomplete (1-6) and complete (7-11) intestinal metaplasia (IM) at the squamocolumnar junction (SCJ)

\begin{tabular}{rlllllll}
\hline No & Age (y) & Sex & $\begin{array}{l}\text { Hiatal } \\
\text { hernia }\end{array}$ & $\begin{array}{l}\text { Erosive } \\
\text { oesophagitis }\end{array}$ & $\begin{array}{l}\text { Histological } \\
\text { oesophagitis }\end{array}$ & $\begin{array}{l}\text { Distal } \\
\text { gastritis }\end{array}$ & $\begin{array}{l}\text { SCJ as reported } \\
\text { visually }\end{array}$ \\
\hline 1 & 33 & $M$ & Yes & No & - & No & Small tongues \\
2 & 35 & $M$ & Yes & Yes & Yes & No & SSBE \\
3 & 37 & $M$ & Yes & No & Yes & No & Tongues $>5 \mathrm{~mm}$ \\
4 & 37 & $F$ & Yes & No & No & No & Tongues $>5 \mathrm{~mm}$ \\
5 & 38 & F & Yes & No & Yes & No & Small tongues \\
6 & 45 & M & No & Yes & No & Yes* & Irritated \\
7 & 22 & M & No & No & Yes & No & Small tongues \\
8 & 24 & $F$ & No & No & No & No & Small tongues \\
9 & 33 & $F$ & Yes & No & No & No & Normal \\
10 & 34 & $F$ & No & No & No & No & Irritated \\
11 & 42 & $F$ & No & No & No & Yes $†$ & Normal \\
\hline
\end{tabular}

*Patient with mild reactive gastritis in the antrum and a normal corpus.

tPatient with autoimmune-type atrophic corpus gastritis and with IM in the corpus.

SSBE, short segment Barrett's oesophagus. 
SCJ, $75 \%$ and $79 \%$ had inflammation at the SCJ. ${ }^{20} 21$ We evaluated patients both with irregular $(50 \%)$ and normal appearing SCJ (50\%); over one third of patients overall (38\%) had inflammation at the SCJ. The lower proportion of cases with inflammation at the SCJ in the current study may be due to the younger age of our patients and inclusion of patients with both irregular and normal appearing SCJs.

In the present study, differences between the two groups were identified in the grade and activity of the inflammatory infiltrate involving columnar mucosa at the SCJ-that is, patients with $H$ pylori associated carditis had a higher severity and activity of inflammation than patients with healthy stomachs. This finding is in agreement with an earlier report $^{20}$ and with a recent study ${ }^{22}$ in which more prominent inflammation and activity were found in cardiac biopsies in $H$ pylori positive patients than in those with GORD.

The congenital or acquired nature of the cardia and histological changes at the SCJ is a matter of continuing debate $^{23-25}$ which takes on added significance given the increase in adenocarcinoma at this region over the last two decades. ${ }^{9}$ The present study is unique in that the study population was limited to young patients in an attempt to minimise the effects of physiological aging factors that may impact on the SCJ. It should be emphasised however that a matching control group of elderly patients was not obtained, thus direct age comparisons could not be made.

Cardiac and fundic mucosa occurred equally often in our biopsy specimens taken at or just below the SCJ. This may reflect the fact that the cardia is a small structure (only millimetres in length), ${ }^{25}$ and that even when sampling close to the SCJ the cardia can be missed (up to $50 \%$ in the present study) and thus only fundic mucosa may be obtained in the biopsy. Moreover, the fact that cardiac mucosa was always present adjacent to squamous oesophageal mucosa-even in young patients with a healthy stomach without symptoms of reflux disease-is further evidence supporting the presence of the cardia as a normal anatomical structure, albeit small, as argued earlier. ${ }^{25}$

In patients with $H$ pylori gastritis, as well as with $H$ pylori negative distal gastritis, both cardiac and fundic mucosa at the SCJ were nearly always inflamed. Instead, in patients with a healthy stomach, cardiac mucosa was inflamed more often than fundic mucosa. In studies comprising patients with GORD, cardiac mucosa at the SCJ was inflamed in $50-96 \%$ of even $H$ pylori negative patients. ${ }^{20}{ }^{26}$ Our patients were relatively young, with only a minority showing signs of GORD. However, among those with a healthy stomach and biopsy specimens including cardiac mucosa, nearly half showed some degree of inflammation at this site. It should be emphasised however that the presence of acid reflux was determined only by patient symptoms prompting endoscopy and not by $\mathrm{pH}$ monitoring, the gold standard for the presence of GORD. Thus the exact prevalence of patients with acid reflux was not known with certainty.

IM is assumed to be a consequence of chronic inflammation. ${ }^{11}{ }^{12}$ Like inflammation at the SCJ, IM at the SCJ has also been associated either with $H$ pylori gastritis or with GORD. ${ }^{27}$ IM in the cardia, as in the gastric antrum, may develop years following active $H$ pylori infection by which time the organisms may be undetectable on biopsy evaluation. In the present study comprising young individuals, only one of 24 patients with $H$ pylori gastritis had IM in the distal stomach, and none had IM at the SCJ. In contrast, IM at the SCJ was demonstrated in one third of patients with a healthy stomach and inflammation at the SCJ. The development of IM at the SCJ was not associated with increased grade and activity of inflammation in this study. This is not surprising given that increased grade and activity of the inflammation was associated with $H$ pylori patients, a group in which not a single case with IM at the SCJ was encountered in this study. This could mean that, possibly due to differing pathogenetic mechanisms in the two types of inflammation, GORD related factors may induce IM at the SCJ earlier than those factors related to $H$ pylori infection.

The histological definition of IM includes the presence of goblet cells; on the basis of the staining pattern of the intermediate non-goblet cells, incomplete and complete types of IM can be distinguished. ${ }^{11}$ Of these, the incomplete type is the more closely associated with cancer. ${ }^{28}$ The fact that Barrett's epithelium is associated with a higher cancer risk than IM in the distal stomach may be due to the more common presence of the incomplete type of IM in Barrett's mucosa. ${ }^{11}$ Incomplete IM at the SCJ has been associated with GORD whereas complete IM has been linked with $H$ pylori gastritis. ${ }^{29}$ However, in our study, as in previous ones, ${ }^{20}{ }^{29}$ approximately half of the patients with a healthy stomach and IM at the SCJ had IM of the complete type. Thus possibly both types of IM may be associated with GORD. That complete metaplasia was found in the youngest individuals in our study raises the question of whether it may precede the development of incomplete IM.

The majority of our $H$ pylori positive patients showed a CagA positive $H$ pylori infection demonstrated by serology. Such an infection has been negatively associated with Barrett's oesophagus. ${ }^{1}$ However, the number of $H$ pylori positive patients in the present study was too small to justify any conclusions as to a possible effect of CagA positive $H$ pylori infection on the development of IM at the SCJ.

The presence of IM at the SCJ has been found to increase with age. ${ }^{29}{ }^{30}$ In a recent Finnish study of a population with a mean age of 57 years, the prevalence of IM at the SCJ in patients with a healthy stomach was $13 \%{ }^{29}$ Among our patients, with a median age of 34 years, $7.2 \%$ of those with a healthy stomach had IM at the SCJ. In patients in whom IM at the SCJ was associated with H pylori gastritis or with IM in the distal stomach, the SCJ often appears macroscopically normal. ${ }^{87}$ In contrast, in patients with IM at the SCJ and signs of GORD, the SCJ may be suggestive of Barrett's oesophagus. ${ }^{8731}$ The correlation between endoscopic findings and the presence of IM may however be poor. ${ }^{6811}$ In this study, $\mathrm{IM}$ at the SCJ was associated with the presence of tongues of mucosa or with tortuosity seen at the SCJ and with negative $H$ pylori status, a profile similar to patients with Barrett's oesophagus; yet only a minority of patients with these findings had IM. The presence of IM may however be patchy, and if only a few specimens are taken the number of patients with IM may be underestimated. In a study which evaluated a large number of consecutive adult patients with a high prevalence rate of $H$ pylori infection and with a normal SCJ, complete cardiac IM was found to be strongly associated with $H$ pylori gastritis. ${ }^{29}$ This is in stark contrast with the present study in which not a single patient with IM at the SCJ was positive for $H$ pylori. The present study shows that when one is evaluating a younger patient population with a low $H$ pylori infection rate and irregular SCJ, IM at the SCJ is less likely to be associated with $H$ pylori infection and more likely to be associated with GORD.

In conclusion, inflammation in the glandular mucosa at the SCJ seems to be either an extension of the multifocal gastritis most often caused by $H$ pylori or an isolated finding, usually milder in nature, associated with signs of GORD and confined mostly to cardiac mucosa. In young individuals, IM at the SCJ seems to be associated with isolated inflammation related to GORD, and not with $H$ pylori gastritis. This may indicate differences in the pathogenesis of inflammation and IM at the SCJ between patients with $H$ pylori infection and those with GORD.

\section{ACKNOWLEDGEMENTS}

This study was supported in part by a grant from the Helsinki University's Research Funds and by Orion Diagnostica, Espoo, Finland. It was presented as a poster at the XIIth International Workshop on 
Gastroduodenal Pathology and Helicobacter pylori in Helsinki, Finland, 11-14 September 2000. We thank Professor Seppo Sarna for kind help with some of the statistical tests and Carolyn Norris, PhD, for skilful aid in revising the English language of the text.

\section{Authors' affiliations}

A Oksanen, Herttoniemi Municipal Hospital, FIN-00099 Helsinki, Finland, and Department of Bacteriology and Immunology, Haartman Institute, University of Helsinki and Helsinki University Central Hospital Laboratory Diagnostics, FIN-00014 Helsinki, Finland

P Sipponen, Department of Pathology, Jorvi Hospital, FIN-02740 Espoo, Finland

R Karttunen, Department of Medical Microbiology, University of Oulu, FIN-90220 Oulu, Finland

H Rautelin, Department of Bacteriology and Immunology, Haartman Institute, University of Helsinki and Helsinki University Central Hospital Laboratory Diagnostics, FIN-00014 Helsinki, Finland

\section{REFERENCES}

1 Vicari JJ, Peek RM, Falk GW, et al. The seroprevalence of cagA-positive Helicobacter pylori strains in the spectrum of gastroesophageal reflux disease. Gastroenterology 1998;115:50-7

2 Weston Ap, Badr AS, Topalovski M, et al. Prospective evaluation of the prevalence of gastric Helicobacter pylori infection in patients with GERD Barrett's esophagus, Barrett's dysplasia, and Barrett's adenocarcinoma. Am J Gastroenterol 2000:95:387-94.

3 Chow W-H, Blaser M, Blot WJ, et al. An inverse relation between $\operatorname{cagA}^{+}$strains of Helicobacter pylori infection and risk of esophageal and gastric cardia adenocarcinoma. Cancer Res 1998;58:588-90.

4 Hansen S, Melby KK, Aase S, et al. Helicobacter pylori infection and risk of cardia cancer and non-cardia gastric cancer. Scand J Gastroenterol 1999; 34:353-60.

5 Blaser MJ. Hypothesis: The changing relationships of Helicobacter pylori and humans: Implications for health and disease. J Infect Dis $1999 ; 179: 1523-30$

6 Spechler SJ, Zeroogian JM, Antonioli DA, et al. Prevalence of metaplasia at the gastro-oesophageal junction. Lancet 1994;344:1533-6.

7 Macdonald CE, Wicks AC, Playford RJ. Ten years' experience of screening patients with Barrett's oesophagus in a university teaching hospital. Gut 1997:41:303-7.

8 Hirota WK, Loughney TM, Lazas DJ, et al. Specialized intestinal metaplasia, dysplasia, and cancer of the esophagus and esophagogastric junction: prevalence and clinical data. Gastroenterology 1999;1 16:277-85

9 Bytzer P, Christensen PB, Damkier P, et al. Adenocarcinoma of the esophagus and Barrett's esophagus: a population-based study. Am J Gastroenterol 1999;94:86-91

10 Conio M, Cameron AJ, Romero Y, et al. Secular trends in the epidemiology and outcome of Barrett's oesophagus in Olmsted County, Minnesota. Gut 2001:48:304-9.

11 Spechler SJ. The role of gastric carditis in metaplasia and neoplasia at the gastroesophageal junction. Gastroenterology 1999;1 17:2 18-28.

12 Nandurkar S, Talley NJ, Martin CJ, et al. Short segment Barrett's oesophagus: prevalence, diagnosis and associations. Gut 1997:40:710-15
13 Johnston $\mathrm{MH}$, Hammond AS, Laskin W, et al. The prevalence and clinical characteristics of short segments of specialized intestinal metaplasia in the distal esophagus on routine endoscopy. Am J Gastroenterol 1996;91:1507-11.

14 Trudgill NJ, Suvarna SK, Kapur KC, et al. Intestinal metaplasia at the squamocolumnar junction in patients attending for diagnostic gastroscopy. Gut 1997;41:585-9.

15 Goldblum JR, Vicari JJ, Falk GW, et al. Inflammation and intestinal metaplasia of the gastric cardia: The role of gastroesophageal reflux and H. pylori infection. Gastroenterology 1998;114:633-9.

16 Ormsby AH, Kilgore SP, Goldblum JR, et al. The location and frequency of intestinal metaplasia at the esophagogastric junction in 223 consecutive autopsies: Implications for patient treatment and preventive strategies in Barrett's esophagus. Mod Pathol 2000;13:614-20.

17 Oksanen A, Sipponen P, Sarna S, Rautelin H. Serologic diagnosis of Helicobacter pylori infection in outpatients aged 45 years or less. Eur $J$ Mirobiol Infect Dis $2001 ; 20: 554-7$.

18 Armstrong D, Bennett JR, Blum AL, et al. The endoscopic assessment of oesophagitis: a progress report on observer agreement Gastroenterology 1966;111:85-92.

19 Dixon MF, Genta RM, Yardley JH, et al. Classification and grading of gastritis. The updated Sydney system. Am J Surg Pathol 1996;20: $1161-81$

20 Bowrey DJ, Clark GWB, Williams GT. Patterns of gastritis in patients with gastro-oesophageal reflux disease. Gut 1999:45:798-803.

21 Voutilainen M, Färkkilä M, Mecklin J-P, et al. Chronic inflammation at the gastroesophageal junction (carditis) appears to be a specific finding related to Helicobacter pylori and gastroesophageal reflux disease. Am J Gastroenterol 1999:94:3175-80.

22 Wieczorek TJ, Wang HH, Glickman J, et al. Clinical-pathologic distinction between reflux carditis and $H$. pylori carditis. Mod Pathol 2002;15:148A.

23 Chandrasoma PT, Lokuhetty DM, Demeester TR, et al. Definition of histopathologic changes in gastroesophageal reflux disease. Am J Surg Pathol 2000;24:344-51.

24 Chandrasoma PT, Der R, Ma Y, et al. Histology of the gastroesophageal junction. An autopsy study. Am J Surg Pathol 2000;24:402-29

25 Kilgore SP, Ormsby AH, Gramlich TL, et al. The gastric cardia: fact or fiction? Am J Gastroenterol 2000;95:921-4

26 Öberg S, Peters JH, DeMeester TR, et al. Inflammation and specialized intestinal metaplasia of cardiac mucosa is a manifestation of gastroesophageal reflux disease. Ann Surg 1997;226;522-32.

27 Hackelsberger A, Gunther T, Schultze V, et al. Intestinal metaplasia at the gastro-oesophageal junction: Helicobacter pylori gastritis or gastro-oesophageal reflux disease? Gut 1998;43:17-21.

28 Filipe MI, Potet F, Bogomoletz WV, et al. Incomplete sulphomucin-secreting intestinal metaplasia for gastric cancer. Preliminary data from a prospective study from three centres. Gut 1985;26:131926

29 Voutilainen $M$, Färkkilä $M$, Juhola $M$, et al. Complete and incomplete intestinal metaplasia at the oesophagogastric junction: prevalences and associations with endoscopic erosive oesophagitis and gastritis. Gut 1999:45:644-8.

30 Aste H, Bonelli L, Ferraris R, et al. Gastroesophageal reflux disease. Relationship between clinical and histological features. Dig Dis Sci 1999;44:2412-18.

31 Eloubeidi MA, Provenzale D. Does this patient have Barrett's esophagus? The utility of predicting Barrett's esophagus at the index endoscopy. Am J Gastroenterol 1999;94:937-43. 